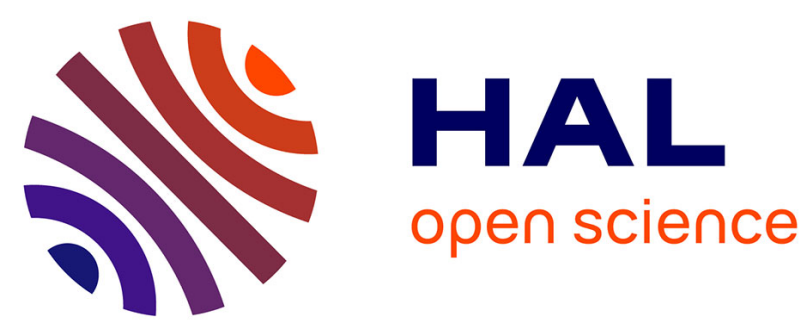

\title{
Anomalous Phonon Behavior: Blueshift of the Surface Boson Peak in Silica Glass with Increasing Temperature
}

\author{
Wolfram Steurer, A. Apfolter, Markus Koch, Wolfgang E. Ernst, Elin
}

Sondergard, Joseph R. Manson, Bodil Holst

\section{- To cite this version:}

Wolfram Steurer, A. Apfolter, Markus Koch, Wolfgang E. Ernst, Elin Sondergard, et al.. Anomalous Phonon Behavior: Blueshift of the Surface Boson Peak in Silica Glass with Increasing Temperature. Physical Review Letters, 2008, 100 (13), pp.135504. hal-00499439

\section{HAL Id: hal-00499439 \\ https://hal.science/hal-00499439}

Submitted on 12 Jul 2010

HAL is a multi-disciplinary open access archive for the deposit and dissemination of scientific research documents, whether they are published or not. The documents may come from teaching and research institutions in France or abroad, or from public or private research centers.
L'archive ouverte pluridisciplinaire HAL, est destinée au dépôt et à la diffusion de documents scientifiques de niveau recherche, publiés ou non, émanant des établissements d'enseignement et de recherche français ou étrangers, des laboratoires publics ou privés. 


\title{
Anomalous Phonon Behavior: Blueshift of the Surface Boson Peak in Silica Glass with Increasing Temperature
}

\author{
W. Steurer,${ }^{1}{ }^{*}$ A. Apfolter,${ }^{1}$ M. Koch,,${ }^{1}$ W.E. Ernst,${ }^{1}$ E. Søndergård, ${ }^{2}$ J.R. Manson, ${ }^{3}$ and B. Holst ${ }^{1, \dagger}$ \\ 1 Institut of Experimental Physics, Graz University of Technology, 8010 Graz, Austria \\ ${ }^{2}$ Laboratoire Surface et Interface du Verre, UMR 125 CNRS/ Saint-Gobain, \\ 39 Quai Lucien Lefranc, 93303 Aubervilliers Cedex, France \\ ${ }^{3}$ Department of Physics and Astronomy, Clemson University, Clemson, South Carolina 29634, U.S.A.
}

(Dated: January 12, 2008)

\begin{abstract}
We present helium atom scattering measurements of the boson peak at the surface of vitreous silica between $127.0 \mathrm{~K}$ and $368.5 \mathrm{~K}$. The most probable energy shows a strong temperature dependence and increases linearly with temperature in the measured range. The observed blueshift of the surface boson peak (shift rate $0.008 \pm 0.002 \mathrm{meV} / \mathrm{K}$ ) is a factor of four to ten times stronger than shift rates measured in the bulk by inelastic neutron and Raman scattering. We suggest that the anomalous shift direction of the boson peak to higher energies with increasing temperature has the same origin as the unusual temperature dependence of the bulk modulus of silica glass.
\end{abstract}

PACS numbers: 61.43.Fs, 63.50.+x,78.30.Ly,72.10.Di

Keywords: Boson peak, vitreous silica, glass

The nature of vibrations in glasses at terahertz frequencies is a question of considerable current interest. A peculiar characteristic of almost all glassy materials is an excess of vibrational states at low frequencies that is not present in their crystalline counterparts $[1,2]$. These modes which are manifest as an excess over the Debye density of states $g(\omega)$, often referred to as the "boson peak" (BP), are closely connected to the plateau that is observed in the specific heat of glasses at a few tens of Kelvin [3]. The BP has been observed by a variety of optical, neutron and thermal measurements [4-7] where a broad peak is seen at terahertz frequencies in $g(\omega) / \omega^{2}$ vs. $\omega$. Recently, the BP has also been observed as a surface phenomenon by inelastic helium atom scattering [8]. In the case of surface sensitive measurements, however, the $\mathrm{BP}$ is most clearly obtained by plotting the surface phonon spectral density divided by $\omega$.

Despite all the progress made in recent years, the nature of the BP is still a matter of ongoing discussions. At the moment there are various theoretical models, none of which can fully explain all aspects simultaneously. Several authors explain the BP on the basis of an acoustic phonon instability [9-12]. Depending on the way the instability is built into the various models, a BP is obtained by modeling a force-constant disorder [9], or, as shown recently, based on the random spatial variation of the shear modulus [10]. Alternatively, the instability has been modeled by studying a topologically disordered structure within Euclidean random matrix theory [11]. The BP has also been described within the phenomenological approach of mode coupling theory [12].

Another popular explanation of the BP which agrees with observations in bulk amorphous silica involves modes resulting from optical-like librations of $\mathrm{SiO}_{4}$ tetrahedra $[4,13]$. However, this model cannot reproduce the anomalous blueshift with increasing temperature pre- sented in this report. Up till now, experimental evidence to prefer one model over the others is lacking.

Whereas a temperature dependence of the intensity of the BP is a well established and universal property of all glasses (in fact, this is how the BP obtained its name) [14-16], the temperature dependence of the most probable energy of the BP differs significantly between materials and is, as a result of this complication, not well understood. In contrast to many glasses which show only a small temperature dependence or where the BP shifts to lower energies with increasing temperature [17, 18], the BP in bulk silica glass shifts to higher energies with increasing temperature (measured shift rates lie between $\approx 2 \cdot 10^{-3} \mathrm{meV} / \mathrm{K}$ and $5 \cdot 10^{-4} \mathrm{meV} / \mathrm{K}$ ) [18-22]. This unusual behavior implies an improbably high negative Grüneisen parameter which has been ascribed to the presence of a positive fourth order anharmonicity in the excess mode potential $[18,20]$. However, the nature of this blueshift with increasing temperature is still awaiting a satisfactory physical explanation.

Here we present measurements of the BP at the surface of vitreous silica that show a much stronger temperature dependence than that of the bulk. The measurements were obtained by inelastic helium atom scattering, a technique which is strictly surface sensitive and does not penetrate into the bulk. Furthermore, the technique has proven to be very well suited for studying surface dynamics [23-25]. The energy of the probing He atoms is in the range of the lowest-energy phonons implying that such measurements are especially sensitive to these modes.

In the course of the experiment time-of-flight (TOF) data of scattered He-particles were recorded for changing surface temperatures. The obtained spectra feature a very sharp and dominant elastic peak and two broad inelastic peak features, one each on the phonon creation 


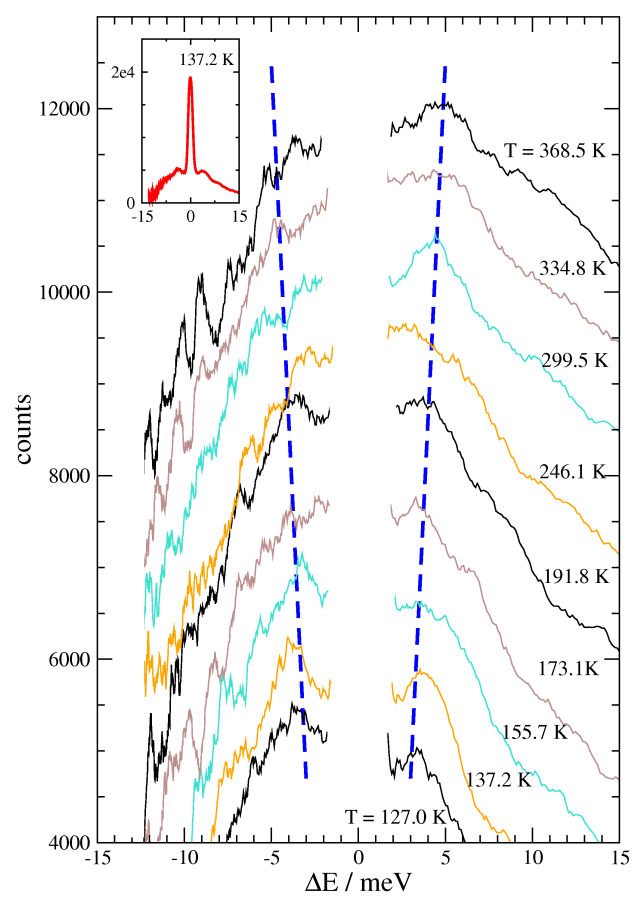

Figure 1: Energy converted TOF spectra as a function of the energy transfer of the He projectile. The most probable energy of the BP shifts to higher energies with increasing temperature and the dashed line is drawn as a guide to the eye. The ordinate depicts the differential reflection coefficient. For the sake of clarity, the elastic peak has been eliminated in all TOFs but an example complete TOF record is shown in the insert for $T=137.2 \mathrm{~K}$. Successive plots are vertically shifted by 1000 counts.

and phonon annihilation sides, respectively, as shown in Fig. 1. The inelastic features are interpreted as the surface manifestation of the BP phenomenon [8]. The succession of spectra clearly reveals the blueshift of the BP with increasing temperature. We attribute the unusual shift direction of the BP in bulk silica glass to the anomalous temperature dependence of the elastic modulus of silica glass which causes a stiffening of the next-nearest neighbor potentials with increasing temperature [26].

The sample used in the present experiments was cut out of polished Spectrosil@ glass and had dimensions of $10 \times 10 \times 1 \mathrm{~mm}^{3}$. The sample was cleaned and annealed in several steps as described in [27] in order to get a smooth surface with properties of a freshly melted glass surface [28]. The experiments were all carried out in the MAGIE apparatus [29] with a base pressure in the $10^{-9}$ mbar range. The surface was exposed to an initial cleaning in situ before starting experiments. A nearly monochromatic beam was created by supersonic expansion with an energy of $19.9 \pm 0.1 \mathrm{meV}$ for all measurements. The scattered helium atoms were ionised by electron bombardment, sent through a magnetic mass selector and detected by a channeltron. The detector entrance was $1618 \mathrm{~mm}$ from the sample surface. The ex- periments presented here were obtained at the specular position $\left(\theta_{i}=\theta_{f}=45^{\circ}\right)$ and the surface temperature was varied from $127.0 \mathrm{~K}$ to $368.5 \mathrm{~K}$. All TOF plots presented herein are converted to an energy-transfer-scale for the abscissa, and to the differential reflection coefficient for the ordinate, respectively. The plots are also corrected for the detection probability of the He atoms which is inversely proportional to the velocity. TOF data was obtained with a bin width of $0.05 \mathrm{~ms}$ and averaged over one hour per spectrum.

The outcome of the experiment is shown in Fig. 1. All TOFs feature a dominant elastic peak (FWHM $\approx$ $1.8 \mathrm{meV}$ ) and some smaller structure which is rather similar in all curves shown. On the phonon annihilation side $(\Delta E>0)$ a broad inelastic peak is seen in all plots. This feature is quite accentuated for some records $(T=137.2 \mathrm{~K}, 299.5 \mathrm{~K}, 368.5 \mathrm{~K})$ while for $T=155.7 \mathrm{~K}$ and $246.1 \mathrm{~K}$ it is less accentuated. Broad peak features are likewise seen on the phonon creation side $(\Delta E<0)$, however, at higher temperatures they become less prominent. This is at least partly due to the higher noise level on the phonon creation side which is an artifact due to the Jacobian transformation used in the conversion of the data from TOF to energy transfer. Only shoulders are seen for some of the records $(T=173.1 \mathrm{~K}$ and $T=299.5 \mathrm{~K})$. Nonetheless, the strong temperature dependence is clearly manifest in the data. With increasing temperature, the observed inelastic features become significantly broader and shift to energies further away from the elastic peak on both the creation and annihilation sides. The dotted lines in Fig. 1 are mirrored about zero energy and trace the trends of the temperature dependence (the slope of the lines is $0.01 \mathrm{meV} / \mathrm{K}$ ). These lines clearly reveal that the features on both phonon creation and annihilation side shift at the same rate.

While the energy resolved spectra presented in Fig. 1 clearly show a strong temperature effect it is important to consider a quantity that has the temperature effects from the Bose-Einstein factors and the Debye-Waller factors removed. For this the surface phonon spectral density, $\rho(\omega, \Delta \underline{K})$ where $\Delta \underline{K}$ is the parallel wavevector, is an ideal quantity to work with for two reasons. Firstly, as was shown in [8], a surface phonon spectral density can readily be extracted from the TOF data. The obtained $\underline{\rho}$ is corrected for all of the factors that are responsible for the asymmetry between the phonon annihilation and creation side in the experiment (such as the Debye-Waller factor and the Bose-Einstein factor). Secondly, as can be seen from Fig. 2, the inelastic features in the TOF (see Fig. 1) appear as excess modes in $\underline{\rho}$ and the surface BP is obtained analogously to the bulk $\overline{\mathrm{BP}}$ via dividing the experimental value by the Debye limit.

A plot of the surface BP as a function of temperature is presented in Fig. 2. In agreement with the behavior of the broad inelastic features in the energy re- 


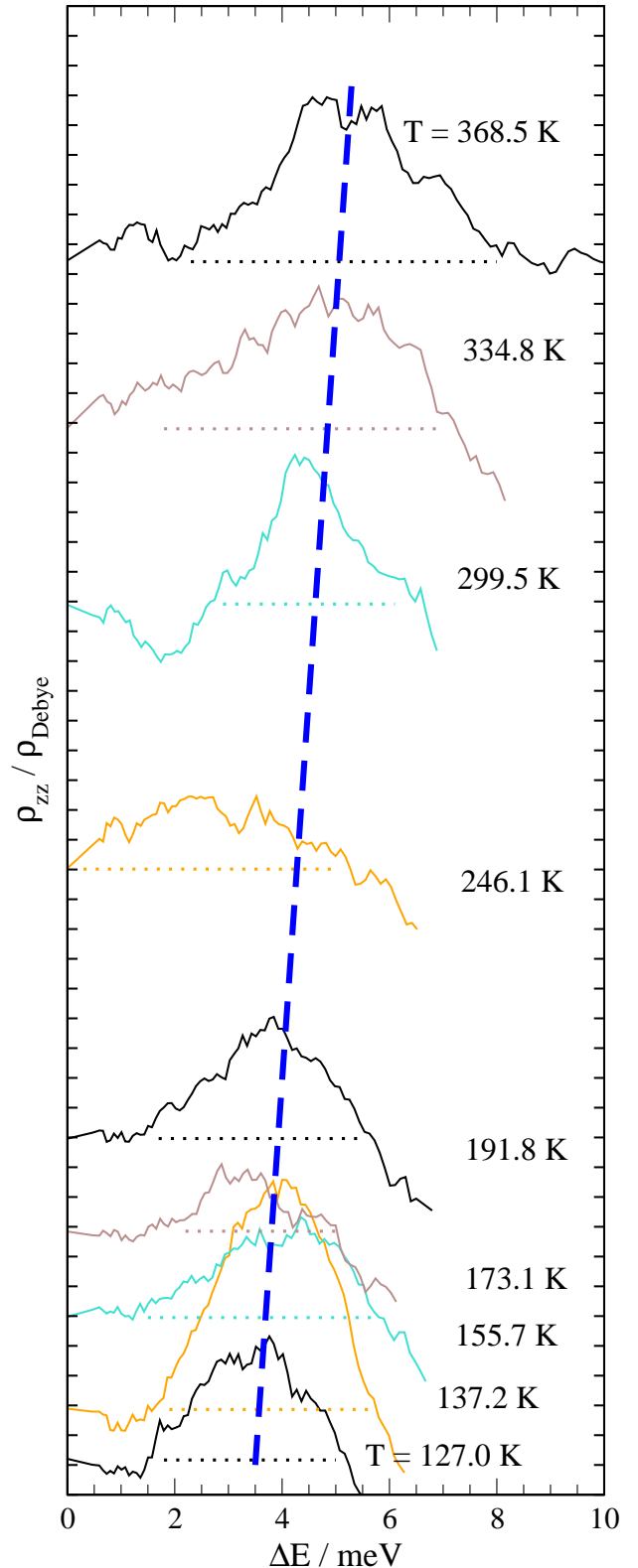

Figure 2: The BP intensity of the surface phonon spectral density divided by the Debye limit, $\rho_{z z} / \rho_{\text {Debye }}$ is shown for the phonon annihilation side of the spectra from Fig. 1 . The most probable energy of the BP exhibits a strong blueshift with increasing temperature whereas the width of the BP does not have a clear temperature dependence. Successive plots are vertically shifted proportional to the temperature $\mathrm{T}$; the dotted lines show $\rho_{z z} / \rho_{\text {Debye }}=1$ for each spectrum (the scale of the ordinate has 0.02 spacing). The dashed line is a guide to the eye (slope $0.008 \mathrm{meV} / \mathrm{K}$ ).

solved spectra presented in Fig. 1, the maximum position of the $\mathrm{BP}$ shifts to higher energies with increasing temperature. However, the observed blueshift of the BP of $0.008 \pm 0.002 \mathrm{meV} / \mathrm{K}$ (as indicated by the dashed line in Fig. 2) is slightly less than what an estimate of the

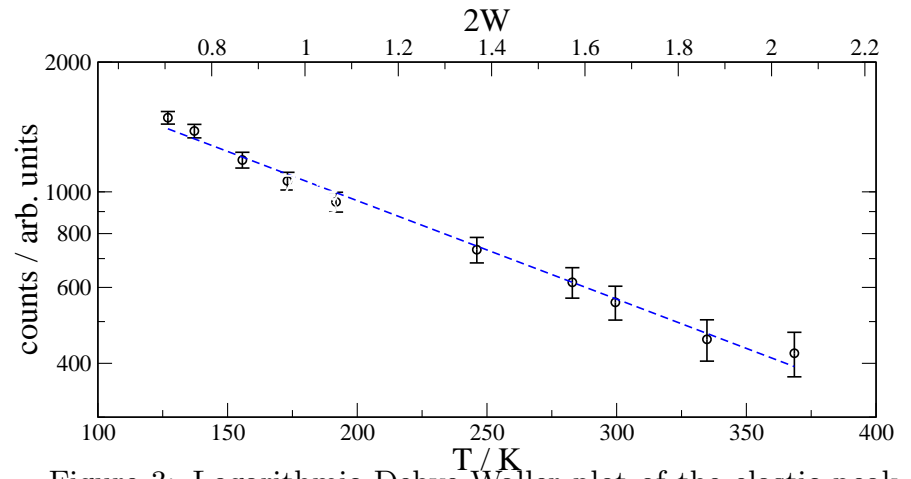

Figure 3: Logarithmic Debye-Waller plot of the elastic peak intensity at the specular position as a function of surface temperature. The incident energy is $19.9 \pm 0.1 \mathrm{meV}$ and the dashed line is an exponential fit to the data. The upper scale on the abscissa gives the value of the Debye-Waller exponent $2 W$.

temperature dependence in the energy converted TOF spectra yields $(\approx 0.01 \mathrm{meV} / \mathrm{K})$. The difference in analyzing the inelastic features from the energy converted TOF spectra (see Fig. 1) and the BP (see Fig. 2) becomes even more apparent when looking at the broadening with increasing temperature. In contrast to the observation made in Fig. 1, no clear temperature dependence of the widths of the $\mathrm{BP}$ is seen in the plots $\rho_{z z} / \rho_{\text {Debye }}$. Thus, the apparent broadening of the inelastic features in the differential reflection coefficient seen in Fig. 1 can be ascribed to the experimental technique.

The extraction of the surface phonon spectral density is based on a theory in the single phonon regime [23], i.e., multiple scattering events must be negligible. Figure 3 shows clearly that for temperatures of order 200 $\mathrm{K}$ or smaller the single-phonon condition is well satisfied and multiple phonon contributions begin to appear only at the highest measured temperatures. If the elastic intensity decays exponentially with increasing temperature and the Debye-Waller exponent is small, then the interaction is clearly in the single-phonon regime since the Debye-Waller exponent roughly corresponds to the number of phonons created or destroyed in a surface collision. That this is the case is shown in Fig. 3 which plots the logarithmic intensity of the elastic peak at the specular position as a function of temperature and as a function of the Debye-Waller argument $2 W$.

The observed temperature dependence of the surface $\mathrm{BP}$ is quite unusual. On the one hand, the BP shifts to higher energies with increasing temperature, and on the other hand, the blueshift observed at the surface is about a factor of four to ten times stronger than that measured in the bulk by Raman scattering [14, 20, 21, 30], inelastic neutron scattering $[18,19,21,30]$, and inelastic x-ray scattering [20] experiments.

The origin of the anomalous shift direction of the BP in bulk silica glass has never been unambiguously clarified 
in the literature. The unusual temperature dependence implies a high negative Grüneisen parameter over a large range up to high temperatures. While the existence of a negative Grüneisen parameter $\gamma$ at low temperatures $(T<15 \mathrm{~K})$ [31] has been known for some time, recent measurements yielded a negative value also at ambient conditions $(\gamma=-1.86)$ [32]. At elevated temperatures, the negative Grüneisen parameter is most probably due to the anomalous temperature dependence of the elastic bulk modulus. This anomalous thermoelastic behavior was observed recently in Brillouin spectroscopy experiments $[33,34]$ and has been qualitatively reproduced by molecular dynamics simulations [26]. Hence, at the highest temperatures considered here which go to just above room temperature the structure of silica glass becomes more rigid than at low temperatures. While for most materials the structure gets softer with increasing temperature, the inverse happens in case of silica glass and, as a consequence, the excess modes shift to higher energies with increasing temperature.

An enhancement of the surface effect compared to that in the bulk is not unusual in case of "normal" anharmonic effects [35] which typically reduce the mode frequency with increasing temperature rather than enhancing it [36-38]. An atom sitting at a surface site is more loosely bound than an atom located in the bulk and is thus more sensitive to variations of the next-nearest neighbor potential. An estimate of the enhancement based on this simple argument yields a factor of two or slightly less compared to the bulk effect, and this is well confirmed for crystalline materials [35]. However, extending this train of argument to the observed anomalous effect only accounts for a fraction of the enhancement of the surface silica BP and cannot satisfactorily explain the anomalously large blueshift. The enhanced shift of the surface BP by a factor of four to ten compared to the bulk BP indicates that the amount of strain at the surface changes more rapidly with temperature than in the bulk. Different kinetic behavior at the surface and in the bulk of silica glass was also observed in relaxation measurements by infrared spectroscopy [39].

In conclusion, the helium scattering data from vitreous silica presented here show an anomalous blueshift of the surface BP with increasing temperature. The observed behavior is unusual, both in terms of direction and strength of the shifting and, furthermore, the shift rate of the surface BP is much stronger than the shift rate of the bulk BP. The anomalous temperature dependence seems to have the same origin as the unusual temperature dependence of the bulk modulus of silica glass. In view of the magnitude of the surface effect, the presented results bear the potential of constituting an important test case for any BP theory.

The authors gratefully acknowledge support from the European Commission, FP6, NEST STREP ADVENTURE program, Project INA, contract number 509014.
* Electronic address: wolfram.steurer@gmail.com

† Present Address: Department of Physics and Technology, University of Bergen, Allegaten 55, 5007 Bergen, Norway

[1] P. Jund and R. Jullien, eds., PHYSICS OF GLASSES: Structure and Dynamics, vol. 489 (AIP Conference Proceedings, 1999).

[2] S. Yannopoulos, K. Andrikopoulos, and G. Ruocco, J. Non-Cryst. Solids 352, 4541 (2006).

[3] T. Nakayama, Rep. Prog. Phys. 65, 1195 (2002).

[4] U. Buchenau, et al., Phys. Rev. B 34, 5665 (1986).

[5] G. Simon, et al., Phys. Rev. Lett. 96, 105502 (2006).

[6] G. Ruocco, et al., Phil. Mag. B 82, 223 (2002).

[7] M. Foret, et al., Phys. Rev. B 66, 024204 (2002).

[8] W. Steurer, et al., Phys. Rev. Lett. 99, 035503 (2007).

[9] S. N. Taraskin, et al., Phys. Rev. Lett. 86, 1255 (2001).

[10] W. Schirmacher, G. Ruocco, and T. Scopigno, Phys. Rev. Lett. 98, 025501 (2007).

[11] T. S. Grigera, et al., Nature 422, 289 (2003).

[12] W. Gotze and M. Sperl, Phys. Rev. Lett. 92, 105701 (2004).

[13] B. Hehlen, et al., Phys. Rev. Lett. 84, 5355 (2000).

[14] G. Winterling, Phys. Rev. B 12, 2432 (1975).

[15] R. Vacher, E. Courtens, and M. Foret, Phys. Rev. B 72, 214205 (2005).

[16] C. Levelut, R. L. Parc, and J. Pelous, Phys. Rev. B 73, 052202 (2006).

[17] Y. Boulmetis, et al., J. Non-Cryst. Solids 347, 187 (2004).

[18] U. Buchenau, et al., J. Physics: Condens. Matter 19, 205106 (2007).

[19] A. Wischnewski, et al., Phys. Rev. B 57, 2663 (1998).

[20] C. Masciovecchio, et al., Phil. Mag. 79, 2013 (1999).

[21] A. Fontana, et al., J. Non-Cryst. Solids 351, 1928 (2005).

[22] A. Kalampounias, S. Yannopoulos, and G. Papatheodorou, J.Non-Cryst. Solids 352, 4619 (2006).

[23] F. Hofmann, J. P. Toennies, and J. R. Manson, J. Chem. Phys. 101, 10155 (1994).

[24] A. Glebov, et al., J. Chem. Phys. 112, 11011 (2000).

[25] J. Lobo, et al.,Phys. Rev. B 74 (2006).

[26] L. Huang and J. Kieffer, Phys. Rev. B 69, 224203 (2004).

[27] W. Steurer, et al., J. Phys.: Condens. Matter, in press.

[28] T. Sarlat, et al., Europ. Phys. J. B 54, 121 (2006).

[29] A. Apfolter, Master's thesis, Graz University of Technology (2005), unpublished.

[30] A. Fontana, et al., Europhys. Lett 47, 56 (1999).

[31] G. K. White, Phys. Rev. Lett. 34, 204 (1975).

[32] R. J. Wang, et al., J. Phys.: Condens. Matter 15, 603 (2003).

[33] A. Polian, D. Vo-Thanh, and P. Richet, Europhys. Lett 57, 375 (2002).

[34] R. Le Parc, et al., J. Phys.: Condens. Matter 18, 7507 (2006).

[35] G. Benedek and J. P. Toennies, Phys. Rev. B 46, 13643 (1992).

[36] H. F. Liu, et al., J. Appl. Phys. 99, 103503 (2006).

[37] J. S. Bae, et al., Phys. Rev. B 73, 052301 (2006).

[38] J. Menéndez and M. Cardona, Phys. Rev. B 29, 2051 (1984).

[39] A. Agarwal and M. Tomozawa, J. Non-Cryst. Solids 209, 264 (1997). 